\title{
Malignant small cell tumour (Askin-Rosai) of the pericardium
}

\author{
K.M. Mohandas ${ }^{1}$, R.F. Chinoy ${ }^{2}$, N.H. Merchant ${ }^{3}$, R.G. Lotliker ${ }^{1}$ and \\ P.B. Desai ${ }^{4}$
}

${ }^{1}$ Critical Care Unit and Departments of ${ }^{2}$ Pathology, ${ }^{3}$ Radiology, ${ }^{4}$ Thoracic and Pediatric Surgery, Tata Memorial Hospital, Parel, Bombay 400 012, India

\begin{abstract}
Summary: An Askin-Rosai tumour presenting as effusive constrictive pericarditis is reported. A 2-D echocardiogram was strongly suggestive of a primary pericardial tumour, and multiple pericardial and pleural aspiration failed to yield malignant cells. The final diagnosis was made at autopsy. Primary pericardial Askin-Rosai tumour has not to our knowledge been previously reported.
\end{abstract}

\section{Introduction}

Primary malignant tumours of the heart and pericardium are extremely rare. ${ }^{1}$ These tumours mimic all the common cardiovascular syndromes and are frequently diagnosed only at autopsy. ${ }^{2}$ Askin et al. have reported a distinctive group of locally invasive, small cell tumours arising in the thoracopulmonary region in children. ${ }^{3}$ A large number of Askin-Rosai tumours have been reported since..$^{4-6}$ We present a primary pericardial Askin-Rosai tumour presenting as effusive-constrictive pericarditis. We believe this to be the first report of a pericardial Askin-Rosai tumour.

\section{Case report}

An 18 year old girl was investigated at another hospital for unresolving haemorrhagic pericardial effusion. Multiple pericardial aspirations did not reveal malignant cells. Her tuberculin test, antinuclear antibodies, and germ cell tumour markers were negative. A computed tomography (CT) scan of the thorax revealed massive pericardial effusion, bilateral pleural effusion and collapse of the left upper lobe. The mediastinum was normal. A bronchoscopy and an ultrasound scan of the abdomen did not reveal any tumour. Empirical therapy for tuberculosis for one month was ineffective. She continued to deteriorate, requiring repeated pleural aspirations and was referred to

Correspondence: K.M. Mohandas, M.D., D.N.B. Accepted: 27 September 1991 our centre.

At admission to our centre she was very ill and had anasarca, tachycardia and tachypnoea. Clinical examination of the heart revealed findings of constrictive pericarditis. Cardiac murmurs and pericardial rub were absent. There was evidence of bilateral pleural effusion, ascites, and a large and tender hepatomegaly. Her chest X-ray revealed cardiomegaly and bilateral pleural effusions. An electrocardiogram revealed sinus tachycardia and generalized $T$ wave inversion. Her previous chest CT scans were not available for review. A two dimensional (2-D) echocardiogram revealed generalized thickening of the pericardium and obliteration of the pericardial cavity with encasement of the heart (Figure 1). The patient died shortly thereafter.

Autopsy revealed haemorrhagic fluid in both pleural cavities with collapsed lungs. The pericardium was greatly thickened by the tumour, measuring up to $5 \mathrm{~cm}$ at places and tightly encasing the whole heart and the root of great vessels (Figure 2). All four cardiac chambers were small and endocardium and valves were normal. Histopathology revealed a monotonous population of small round cells without mitosis or necrosis. The tumour infiltrated the myocardial fibres and both pulmonary hila. Immunohistochemical staining for cytokeratin, leucocyte common antigen, epithelial membrane antigen, and vimentin were negative. No other primary tumour or metastatic deposits were seen in the thorax and abdomen. A diagnosis of malignant small cell tumour of childhood of the thoraco-pulmonary region (Askin-Rosai tumour ${ }^{5}$ ) was made. 


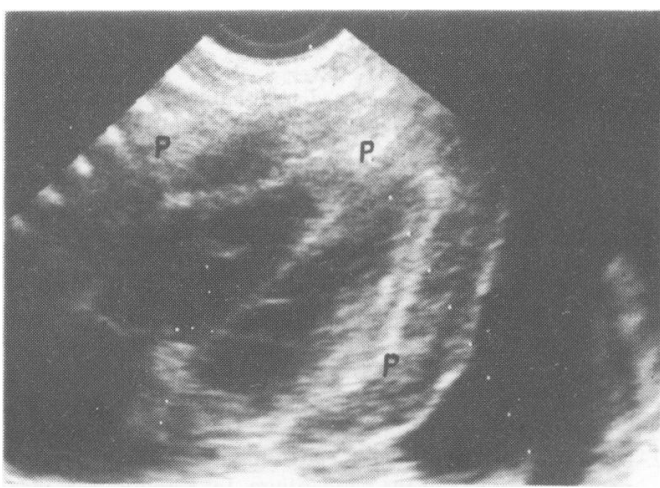

Figure 1 2-D echocardiogram showing thickened pericardium (P) obliterating the pericardial cavity and totally encasing the heart.

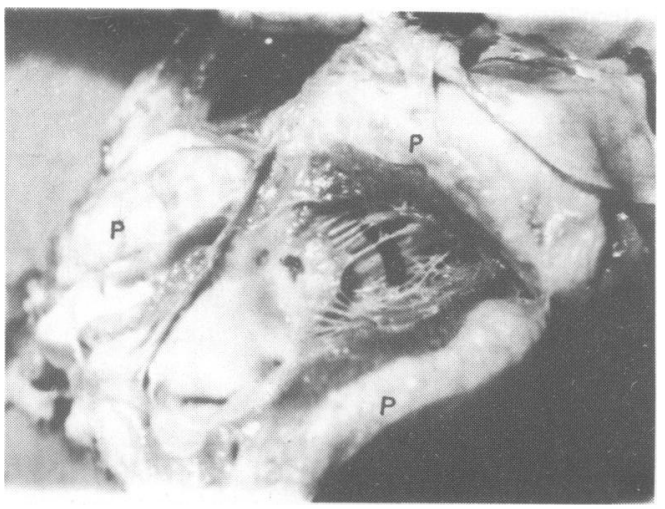

Figure 2 Cut open specimen of the heart showing thickened pericardial tumour (P) encasing the heart.

\section{Discussion}

Askin-Rosai tumours are uncommon childhood tumours, and have not been reported to arise in the pericardium. Our patient with pericardial AskinRosai tumour presented initially with haemorrhagic pericardial effusion which subsequently evolved into a constrictive pericardial tumour. Common childhood tumours such as lymphoid, gonadal, hepatic renal and pulmonary tumours were excluded. As multiple pericardial and pleural aspirations failed to reveal malignant cells a primary pericardial tumour was not diagnosed until autopsy.

A 2-D echocardiogram performed later in the illness revealed circumferential pericardial thickening, which was suggestive of a pericardial tumour. The complexity of many pericardial diseases and the risk of pericardial surgery require a multimodality imaging approach for complete definition. ${ }^{7}$ 2-D echocardiography is the primary imaging modality. ${ }^{7-9} \mathrm{~A}$ consistent finding of pericardial tumours on 2-D echocardiogram is localized or generalized thickening of the pericardium as seen in our patient, which can also occur in infective, fibrous, granulomatous disorders. ${ }^{10}$ Chronic effusion of any aetiology may develop fibrous strands that can be mistaken for tumours. ${ }^{10}$ Hence it is necessary to establish a histopathological diagnosis. Simple aspiration cytology may be ineffective. Multiple biopsies of the pericardium can be safely obtained by new techniques. ${ }^{11}$

CT scan is currently the investigation of choice in the pre-surgical evaluation of pericardial tumours. ${ }^{7,12}$ It can differentiate encysted effusions from masses and the extension of the tumour into extrapericardial structures. ${ }^{7,12} \mathrm{CT}$ scan also aids in guided biopsies of localized pericardial masses. ${ }^{7,12}$ The CT scans performed early in the illness of our patient had revealed massive pericardial effusion, but were not available for a retrospective review. Magnetic resonance imaging (MRI) is now being evaluated in imaging pericardial tumours. ${ }^{7,9,12-14}$ Advances in MRI technology and gating to cardiac cycle can demonstrate the vessel and mass relationship and the intra- and extra-cardiac extensions of the tumours. ${ }^{14}$ MRI can differentiate between tumours and other inflammatory disorders based on tissue characteristics, and guide to the appropriate site for biopsy. ${ }^{14}$

Common primary tumours of the heart and the pericardium in children include teratocarcinoma, rhabdomyosarcoma, neurogenic sarcoma and fibrosarcomata. ${ }^{1}$ Ours is the first report of an Askin-Rosai tumour affecting the pericardium. These small cell tumours occur in the thoracopulmonary region of children and rarely metastasize to distant areas as seen in our patient. Askin-Rosai tumour is often an exclusion diagnosis not always readily made and generally presents in the second decade with a female predilection. ${ }^{4,5}$ The best results on the treatment of these tumours are by complete surgical resection; radiotherapy and current chemotherapy are not curative. ${ }^{4,6}$ Our patient at initial presentation had involvement of the whole pericardium and pleura manifesting as haemorrhagic effusions. Hence curative resection would not have been possible.

We conclude that early diagnosis and treatment of pericardial neoplasms can be achieved only by a high index of clinical suspicion. The presence of thickening of the pericardium on a 2-D echocardiogram in the absence of a definite aetiology warrants an early pericardial biopsy for the diagnosis of pericardial tumours in an early stage.

\section{Acknowledgement}

We thank Dr P.G. Isaacson and Dr A.J. Norton of the Department of Histopathology, School of Medicine, University College London, UK, for reviewing the pericardial histopathology. 


\section{References}

1. McAllister, H.A. Jr. \& Fenoglio, J.J. Jr. Tumours of the cardiovascular system. Pathol Annu 1979, 14: 325-355.

2. Hall, R.J. \& Cooley, D.A. Neoplastic heart disease. In: Hurst, J.W. (ed) The heart, arteries and veins. McGraw Hill, New York, 1982, pp. 1403-1424.

3. Askin, F.B., Rosai, J., Sibley, R.K., Dehner, L.O. \& McAllister, W.H. Malignant small cell tumour of the thoraco-pulmonary region in childhood. A distinctive clinicopathological entity of uncertain histogenesis. Cancer 1979, 43: 2348-2351.

4. Bourque, M.D., Di Lorenzo, M., Collin, P.P., Russo, P., Laberge, J.J. \& Moir, C. Malignant small cell-tumour of the thoracopulmonary region; Askin tumour. J Pediatr Surg 1989, 24: 1079-1083.

5. Coffin, C.M. \& Dehner, L.P. Peripheral neurogenic tumours of the soft tissues in children and adolescents: A clinicopathologic study of 139 cases. Pediatr Pathol 1989, 9: 387-407.

6. Parikh, P.M., Charak, B.S., Banavali, S.D. et al. Treatment of Askin Rosai tumour: Need for a more aggressive approach. J Surg Oncol 1988, 39: 126-128.

7. Miller, S.W. Imaging pericardial disease. Radiol Clin North Am 1989, 27: 1113-1125.
8. Kotler, M.N. Metastatic cardiac tumours: recognition of pericardial, myocardial and endocardial involvement by two dimensional echocardiography. In: Kapoor, A.S. (ed) Cancer and the heart. Springer Verlag, New York, 1986, pp. 51-61.

9. Rienmuller, R., Lloret, S.L., Tiling, R. et al. MR Imaging of pediatric cardiac tumours previously diagnosed by echocardiography. J Comput Assist Tomogr 1989, 13: 621-626.

10. Roberts, W.C. \& Spray, T.L. Pericardial heart disease: A study of its causes, consequences and morphological features. Cardiovasc Clin 1976, 7: 11-65.

11. Endrys, J., Simo, M., Shafie, M.Z. et al. New non-surgical technique for multiple pericardial biopsies. Cathet Cardiovasc Diagn 1988, 15: 92-94.

12. Moncada, R. \& Posniak, H. Computed tomography of neoplastic disease of the pericardium. In: Kapoor, A.S. (ed) Cancer and the heart. Springer Verlag, New York, 1986, pp. 26-41.

13. Lund, J.T., Ehman, R.L., Julsrud, P.R., Sinak, L.J. \& Tajik, A.J. Cardiac masses: assessment by MR imaging. AJR 1989, 152: 469-473.

14. Templeton, P.A. \& Zerhouni, E.A. MR Imaging in the management of thoracic malignancies. Radiol Clin North Am 1989, 27: 1099-1111. 